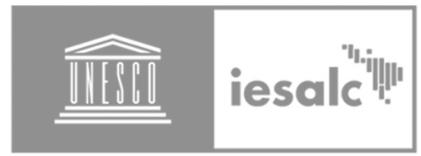

\title{
Unesco
}

Instituto Internacional

para la Educación Superior

en América Latina

y el Caribe
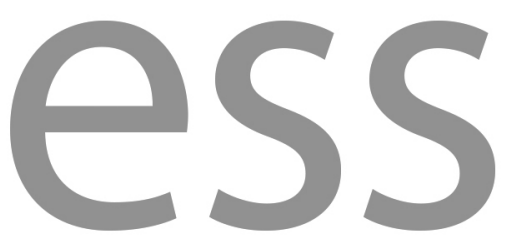

?

Educación

Superior y

Sociedad

Vol. 33 No. 2 (2021)

-

$\bullet$

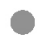

$\bullet$
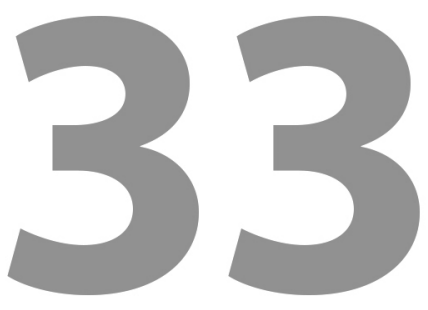

Incluye Dossier Temático:

Desafíos de la educación superior frente a la pandemia de Covid-19 en América Latina y el Caribe 


\section{Contribución de las universidades argentinas públicas al logro del ODS sobre la igualdad de género y empoderamiento de las mujeres y las niñas (2015-2019)}

Mariana Laura Solans

\section{RESUMEN}

El presente artículo explora desde una perspectiva analítica y crítica las contribuciones de las universidades argentinas públicas al logro del Objetivo de Desarrollo Sostenible (ODS) sobre la igualdad de género y empoderamiento de las mujeres y las niñas, y a la meta de eliminar todas las formas de discriminación y violencia en contra de ellas. Para ello, se hace referencia al proceso de incorporación de las mujeres en el ámbito de la Educación Superior en Argentina y a los obstáculos planteados en el reconocimiento de sus derechos; así como al desarrollo temprano de las ideas feministas y su vinculación con la vida universitaria. Estos elementos son significativos para mostrar las características del impulso dado a partir de 2015 y desde abajo hacia arriba, mediante la labor de las feministas y movimientos de mujeres, al logro de este Objetivo por parte de las universidades. Sin embargo, dichos procesos y aportes no fueron suficientemente reconocidos en el campo de las políticas públicas contenidas en la materia y en el período de referencia.

Palabras clave: ODS; Naciones Unidas; Universidades Nacionales; Igualdad de género; Género y Educación.

\section{Contribution of Argentine public universities to the achievement of the SDG: gender equality and all women's and girls' empowerment (2015-2019)}

\section{ABSTRACT}

The paper explores, from an analytical and critical perspective, the contribution of Argentine public universities to the achievement of the UN's Sustainable Development Goal (SDG) related to gender equality and all women's and girls' empowerment, and to the elimination of all forms of discrimination and violence 
against them. In order to do it, the paper analyses the process of incorporation of women into higher education in Argentina and the obstacles they have to endure to obtain the recognition of their rights; as well as the early development of the feminist ideas in the country and their connection to the university's life. To achieve this SDG in the universities, it was necessary the impulse provided by a bottom-up movement since 2015, composed by the hard work of feminist and women's movements. However, these processes and contributions were not sufficiently acknowledged by the nation state in the implementation of public policies for this sector during the time convered by this paper.

Keywords: SDG; United Nations; Public Universities; Gender Equality; Gender and Education

\section{Contribuição das universidades públicas argentinas para o ODS sobre igualdade de gênero e empoderamento de mulheres e meninas (2015-2019)}

\section{RESUMO}

Este artigo explora desde uma perspectiva analítica e crítica as contribuições das universidades públicas argentinas no cumprimento do Objetivo de Desenvolvimento Sustentável (ODS) sobre igualdade de gênero e empoderamento de mulheres e meninas, e a eliminação de todas as formas de discriminação e violência contra elas. $O$ processo de inclusão das mulheres no âmbito da educação superior na Argentina, os obstáculos enfrentados no reconhecimento de seus direitos; junto com o desenvolvimento do ideário feminista e a relação deste com a vida universitária foram elementos referenciados. Para alcançar esse objetivo nas universidades, o impulso gerado desde 2015 pelo trabalho das feministas e o movimiento de mulheres foi necessário. Porém, estes processos e aportes não foram reconhecidos nas políticas públicas citadas no artigo nem no período de referência considerado.

Palavras chave: ODS; Nações Unidas; Universidades; lgualdade de Gênero; Gênero e Educação. 


\section{Contribution des universités publiques argentines à la réalisation de l'ODD sur l'égalité entre les sexes et l'autonomisation de toutes les femmes et les filles (2015-2019)}

\section{RESUMÉ}

Cet article explore d'un point de vue analytique et critique les contributions des universités publiques argentines à la réalisation de l'Objectif de Développement Durable (ODD) sur l'égalité entre les sexes et l'autonomisation des femmes et des filles, et à l'élimination de toutes les formes de discrimination et de violence à leur encontre. Pour cela le travail fait référence au processus d'intégration des femmes au sein de l'enseignement supérieur en Argentine et aux obstacles soulevés à partir de la reconnaissance de leurs droits; ainsi que le développement précoce des idées féministes et leur lien avec la vie universitaire. Afin d'atteindre cet objectif au sein des universités, une impulsión générée par un mouvement massif ascendant à partir de 2015 a été nécessaire, composée par le travail des féministes et les mouvements de femmes. Cependant, ces processus et contributions n'ont pas été suffisamment reconnus dans le domaine des politiques publiques au cours de la période de référence.

Mots clés: ODS; Les Nations Unies; Universités Nationales; Égalité entre les Sexes; Genre et Éducation.

\section{INTRODUCCIÓN}

El presente artículo explora desde una perspectiva analítica y crítica las contribuciones de las universidades argentinas públicas al logro del ODS sobre la igualdad de género y empoderamiento de las mujeres y las niñas, y a la meta de eliminar todas las formas de discriminación y violencia en contra de ellas. Para ello, se hace referencia al proceso de incorporación de las mujeres en el ámbito de la Educación Superior en Argentina y a los obstáculos planteados en el reconocimiento de sus derechos; así como al desarrollo temprano de las ideas feministas y su vinculación con la vida universitaria. Estos elementos son significativos para mostrar las características del impulso dado a partir de 2015 y "desde abajo hacia arriba", mediante la labor de las feministas y movimientos de mujeres, al logro de este Objetivo por parte de las universidades. 
Este artículo sostiene que, pese al protagonismo de las universidades durante el período analizado en la promoción de la institucionalización y transversalización de la perspectiva de género en el ámbito de sus funciones, no fueron suficientemente consultadas, ni su rol adecuadamente reconocido, por los organismos nacionales responsables de las políticas públicas de género y diversidad. No obstante ello, generaron una red de trabajo, participación y debate que hoy en día permite la apertura de un espacio transformador, tanto en el ámbito de las propias instituciones universitarias como en su articulación con el medio.

\section{Incorporación de las mujeres a la educación superior en Argentina}

En Argentina, el derecho a la educación fue jurídicamente consagrado para todos los habitantes desde la Constitución Nacional sancionada en 1853. Las reformas posteriores explicitaron para las mujeres, la garantía de la igualdad real de oportunidades y de trato, y el pleno goce y ejercicio de los derechos reconocidos por la Constitución y por los tratados internacionales vigentes sobre derechos humanos (Constitución de la Nación Argentina, 1994, Art. 75).

Como parte de este proceso, la sanción de la primera Ley de Educación del Estado Nacional emergente, estableció la instrucción primaria obligatoria, gratuita y gradual (Ley $\left.N^{\circ} 1.420,1884\right)$. La escuela pública se puso al alcance de todos los niños y niñas, definiéndose un mínimo de instrucción obligatoria, a la que se sumaron, en el caso de los varones, conocimientos sobre ejercicios militares, nociones de agricultura y ganadería, y para las niñas, aquellos referidos a labores de manos y economía doméstica. De esta manera, la Ley asignó tareas y esferas de actuación diferenciadas para hombres y mujeres. Esta división social del trabajo, a partir de la cual las mujeres fueron relegadas al ámbito de lo privado y los hombres a la esfera de lo público, profundizó relaciones sociales de poder y de dominación entre los géneros que persisten al presente.

La sanción de la Ley de Educación N ${ }^{\circ} 1.420$ generó, además, una demanda de formación de maestros para darle cumplimiento y llevó a la creación de las primeras Escuelas Normales. En este contexto, las mujeres fueron convocadas a asumir el rol de educadoras y fueron reconocidas como trasmisoras de la ideología del Estado Nacional de finales del siglo XIX que se estaba gestando. El ejercicio del magisterio fue percibido como una prolongación de las funciones y rasgos maternales asociados a la mujer, y dotado de un cuerpo docente femini- 
zado, de bajo costo y precarizado. No obstante, la introducción de estas escuelas habilitó para ellas un espacio de ejercicio profesional fuera del hogar, preparó el camino para su reclamo de estudios universitarios y significó una primera intervención legítima en el ámbito público(1). Las contradicciones emanadas de este contexto se manifestaron a través de debates en torno a la educación femenina y los contenidos de la enseñanza; a los que se sumaron noticias referidas a la incorporación de mujeres a las universidades en otros países, en carreras fundamentalmente relacionadas con las ciencias de la salud (Palermo, 2005).

Al momento de la sanción de la Ley $N^{\circ} 1.420$, existían en nuestro país dos universidades, la Universidad Nacional de Córdoba y la Universidad de Buenos Aires, y cuatro orientaciones de estudio agrupadas en torno a facultades: Medicina, Derecho, Filosofía y Letras, y Ciencias Exactas, Físicas y Naturales. Estas instituciones ofrecían carreras superiores, como Medicina y otras menores como Obstetricia, Odontología y Farmacia. Es a través de estas carreras, que las mujeres encontraron su puerta de acceso a la universidad. La aceptación por parte de la sociedad de su lenta y acotada incorporación en estas áreas de estudio, denotó una vez más la vigencia de las funciones de cuidado de otros y otras asignadas a la mujer como tal. Pero de acuerdo con lo expresado por Palermo (2006), aún en el campo de la Medicina, debieron enfrentar obstáculos en torno a qué espacios profesionales se consideraban vedados para ellas, por ejemplo, la docencia universitaria o la cirugía. Seguidamente, la creación a finales del siglo XIX de la Facultad de Filosofía y Letras, y la posibilidad de ingresar a ella con el requisito del título de maestra normal, marcó una reorientación en la elección femenina de los estudios universitarios.

\section{Desarrollo de las ideas feministas y su vinculación con la vida universitaria}

Las primeras tres décadas del siglo XX, aún con pocas mujeres en las aulas universitarias, mostraron su participación en debates, congresos, publicaciones, desarrollando un importante movimiento a favor de las demandas feministas. De acuerdo con lo expresado por Palermo "con las primeras mujeres que accedieron a la Universidad se inició en nuestro país una línea de preocupación teórica y práctica que constituye un claro antecedente de los estudios de edu-

(1) Véase: Anzorena, C. (2008). 
cación de género" (2006, p. 42). La reivindicación por la educación de las mujeres constituía una preocupación del movimiento feminista en general y del debate de la época. En 1910, la "Asociación Universitarias Argentinas" convocó al Primer Congreso Femenino Internacional, donde se debatió el papel de las mujeres y su relación con la Sociología, el Derecho, la Educación, las ciencias, las letras, las artes y las industrias (Gomez, 2019). Cuando en 1918 estalló en la Universidad Nacional de Córdoba la Reforma Universitaria, ya había numerosas estudiantes y graduadas mujeres, así como agrupaciones que reclamaban la igualdad entre los sexos. Pese a que el reclamo estudiantil iniciado apeló a la democratización de la educación y marcó un hito en la Educación Superior al establecer la autonomía universitaria y el cogobierno, las reivindicaciones en torno a la eliminación de los obstáculos para la educación de las mujeres, no fueron contempladas en el marco de dicha reforma (Bustelo, 2018). Para la década de 1920, se multiplicaron los grupos de mujeres que demandaron derechos, especialmente en torno al reconocimiento civil y político. En 1926 se sancionó la primera reforma del Código Civil, a partir de la cual las mujeres alcanzaron la igualdad legal con los varones, aunque se refiere a una igualdad relativa por no incluir la patria potestad compartida ni el derecho al voto. En los años siguientes, frente al avance de los regímenes totalitarios, la acción feminista en procura de los derechos sufrió una mengua. En 1947, durante el gobierno de Juan Domingo Perón y de la mano de su esposa, Eva Perón, se promovió la sanción del acceso de las mujeres al sufragio. Pero la caída del peronismo mediante un golpe de estado en 1955, y el nuevo golpe de 1976, inauguraron un ciclo de represión, en el que algunos núcleos feministas pudieron sostenerse tímidamente (Barrancos, 2012).

A partir de la recuperación democrática en 1983, resurgieron con especial vigor diversas expresiones feministas que confluyeron en un avivamiento de las luchas por derechos y demandas de equidad. Es así como a inicios de la década del ochenta del siglo XX, dos instituciones iniciaron el desarrollo de estudios sistemáticos sobre algunos aspectos de la condición femenina: el Centro de Estudios del Estado y la Sociedad (CEDES) y el Centro de Estudios de Población (CENEP). Sus indagaciones cimentaron la base de los estudios que luego se expandirían. En 1987, la Facultad de Psicología de la Universidad de Buenos Aires inició el ciclo de una Carrera Interdisciplinaria de Estudios de la Mujer, que significó la primera incorporación de los estudios a la vida universitaria argentina. 
En la década siguiente surgieron en diversas universidades nacionales centros, áreas, programas dedicados a la investigación de la condición femenina y las relaciones de género que se plasmaron esencialmente en la formación de estudiantes de cursos de posgrado. Es así como el siglo XXI, se abrió con una vasta producción de estudios e investigaciones que contribuyeron en el marco del sistema universitario y científico a los estudios de las mujeres y de género (Barrancos, 2012).

\section{Contribución de las universidades nacionales al logro de la igualdad de género}

Si bien el desarrollo de las ideas feministas y el involucramiento de las universitarias en la problemática de las mujeres data de principios del siglo pasado, en los últimos tiempos se ha asistido en Argentina a un proceso inédito de instauración de políticas de género en las Instituciones de Educación Superior. La aparición hacia finales del siglo XX de los estudios de las mujeres y de género, y de los distintos espacios dedicados a la investigación de la temática, crearon las condiciones para que en la segunda década del siglo XXI (más puntualmente desde el año 2014, con la sanción del primer Protocolo de actuación contra la violencia de género( ${ }^{(2)}$ se inicie un proceso de profundización y ensanchamiento de las políticas de género en las universidades públicas del país (Torlucci et al., 2019).

(2) El Protocolo de intervención institucional ante denuncias por situaciones de violencia sexista, estableció que dichas denuncias se realizarán en la Secretaría de Bienestar Universitario, donde serán tomadas por personas capacitadas para ello, que brindarán asesoramiento para tramitar apoyo psicológico a la/s persona/s denunciante/s. Se dejó constancia, asimismo, de que en el supuesto de que los hechos que dan motivo a la denuncia recibida pudieran constituir un ilícito, los funcionarios están obligados a denunciarlos a la justicia. Las acciones objeto del presente protocolo serán consideradas faltas graves, a los efectos de los procedimientos de investigación y regímenes sancionatorios correspondientes al personal docente y no docente. Para los estudiantes y todo otro integrante de la comunidad universitaria no comprendido en las categorías anteriores, se propondrán -a través del Consejo Directivo- las medidas de sanción, que posteriormente se elevarán al Consejo Superior para su resolución. El área que recibe la denuncia deberá remitirla al Decano o Decana de la Unidad Académica, o autoridad correspondiente, en un plazo máximo de dos días hábiles. 
Dicho proceso cobró fuerza a partir de dos hechos relevantes. Por un lado, la convocatoria realizada por un colectivo de voluntades feministas en junio de 2015 a una primera movilización bajo la consigna "Ni Una Menos" (Ni Una Menos, 2015), con objeto de visibilizar la violencia machista, tras el femicidio de Chiara Páez ${ }^{(3)}$. Por otro lado, el femicidio de Micaela García en abril de $2017^{(4)}$, a partir del cual se impulsó la Ley № 27.499 que lleva su nombre. Como parte de sus fundamentos, el proyecto señalaba el incremento de los femicidios en los últimos años y la posibilidad de evitarlos de haber existido una eficiente intervención de los agentes del Estado, en sus distintos niveles y jerarquías (Cámara de Diputados, 1776-D-2017). Tras un intenso debate que atravesó a la sociedad, el día jueves 10 de enero de 2019 se publicó en el Boletín Oficial la sanción de la "Ley Micaela de capacitación obligatoria en género para todas las personas que integran los tres poderes del Estado".

El impacto de estos sucesos en las universidades se visibilizó, a través de la creación en el año 2015, de la Red Interuniversitaria por la lgualdad de Género y Contra las Violencias (RUGE)(5), que se institucionalizó como tal en 2018, en el

A partir de allí, en un plazo no mayor a cinco días hábiles, dicha autoridad notificará a la persona denunciada, quien presentará un descargo por escrito en el término de dos días hábiles; elaborará el informe correspondiente; y convocará a una sesión extraordinaria del cuerpo colegiado cuando correspondiere (Ordenanza del Consejo Superior de la Universidad Nacional del Comahue № 1572, 2014).

(3) Chiara Páez era una adolescente de 14 años, brutalmente asesinada a golpes por su novio, Manuel Mansilla. Al momento de su asesinato, Chiara Páez se encontraba embarazada.

(4)Micaela García, de 21 años, fue secuestrada, violada y asesinada por Sebastián Wagner, quien había sido condenado a nueve años de prisión por abuso sexual, y contaba con el beneficio de la excarcelación.

(5)La Red empezó a gestarse en las Jornadas de Historia de las Mujeres en la provincia de Neuquén, en marzo de 2015. Se concretó y formalizó el día 3 de septiembre de 2015 en el campus Migueletes de la Universidad Nacional de San Martín, con la presencia de docentes, investigadoras, estudiantes y autoridades de 25 universidades argentinas. 
marco del Consejo Interuniversitario Nacional $(\mathrm{CIN})^{(6)}$. A través de su pronunciamiento en la III Conferencia Regional de Educación Superior, la Red convocó a las universidades públicas del país a comprender que una sociedad más justa e igualitaria, es una sociedad que se piensa y se desea a sí misma libre de cualquier opresión de género y proponiendo la ampliación de las pluralidades de los sujetos que la componen (RUGE, 2018). Seguidamente, las instituciones que conforman el CIN formalizaron su adhesión a la Ley Micaela, recomendando el desarrollo y la promoción de políticas tendientes a erradicar la desigualdad entre los géneros. Se encomendó a RUGE su actuación como órgano de consulta, capacitación permanente y asesoramiento en las temáticas de su competencia, mediante una asistencia confidencial y no vinculante; y se acordó promover acciones destinadas a la creación y fortalecimiento de protocolos (CIN, 2019).

De acuerdo con lo expresado por Torlucci et al. (2019), desde el año 2013, a través del activismo de docentes, investigadoras, no docentes, estudiantes y decanas, se inició un proceso de discusión y creación de protocolos y programas en las universidades para dar respuesta a situaciones de discriminación y violencia de género vividas dentro de las instituciones. Como parte de este proceso, en 2014, la Universidad Nacional del Comahue fue la primera en aprobar su Protocolo de intervención institucional ante denuncias por situaciones de violencia sexista en el ámbito de la universidad (Ordenanza CS N 1572/14).

En el estudio elaborado por Torlucci et al. (2019), se detalla, asimismo, que para 2019 eran 45 las universidades que contaban con protocolos, representando el $73,8 \%$ del sistema universitario. Mientras que el $19,7 \%$ de las instituciones universitarias restantes se encontraban diseñando y elaborando los proyectos de protocolo para ser aprobados próximamente. Desde 2015, la cantidad de universidades que aprobaron estas herramientas aumentaron de forma continua, del $9 \%$ del total en 2015 al casi 30\% en 2017. Entre 2017 y 2018 se advirtió un incremento relacionado con la acumulación de experiencia y trabajo político de

${ }^{(6)}$ El Consejo Interuniversitario Nacional fue creado por Decreto del Presidente de la República Argentina, Dr. Raúl Alfonsín, el 20 de diciembre de 1985. El CIN tiene funciones de coordinación, consulta y propuesta de políticas y estrategias de desarrollo universitario y la promoción de actividades de interés para el sistema público de Educación Superior. Es, además, órgano de consulta obligada en la toma de decisiones de trascendencia para el sistema universitario. 
las feministas universitarias, sumado al proceso de movilizaciones que "tuvo un claro auge en 2015 con el primer Ni Una Menos, luego el paro internacional de mujeres y en 2018 los pañuelazos a partir del debate en el Congreso de la Nación sobre la legalización y despenalización del aborto" (ob.cit., p. 4). La adhesión del CIN a la Ley Micaela y su proceso posterior de legitimación por parte de sus consejos superiores terminó de impactar sobre las universidades que aún no tenían protocolos. Cada institución definió las características y objeto de su herramienta, así como las medidas tendientes a la prevención, atención, sanción y ámbito de aplicación. Dependiendo de los casos, supieron contemplar la actuación ante situaciones de discriminación, violencia, agresión, hostigamiento, acoso sexual; basadas en cuestiones de género (identidad o expresión de género), orientación sexual, vivencia de la sexualidad, violencia hacia las mujeres y/o a la comunidad LGBTIQ+ (Lesbianas, Gays, Bisexuales, Transgénero, Travesti, Intersexual, Queer, y otras).

La incorporación de los referidos conceptos y la implementación de los dispositivos, pusieron en evidencia la necesidad de crear espacios de género institucionalizados, que garantizaran su aplicación, para luego dar el paso a la transversalización institucional de la temática. En tal sentido, los datos provistos por Torlucci et al. (2019) mostraron en 2019 , que casi el $60 \%$ de las universidades contaban con un espacio institucionalizado dedicado a cuestiones de género, de los cuales el 55,2\% tenía rango de "Programa", con muestras de crecimiento y evolución, a partir de la creación de secretarías, sub-secretarías y direcciones como espacios de mayor jerarquía. Desde la adhesión del CIN a la Ley Micaela, el $81,9 \%$ de las universidades ratificaron su adhesión a la norma. Del total de instituciones universitarias, el 47,5\% inició para 2019 las capacitaciones en los distintos claustros que componen las universidades; $31 \%$ con las autoridades; $27 \%$ al claustro no docente; y el $21 \%$ al claustro docente y estudiantil (ob.cit.).

Es importante destacar que las distintas acciones consignadas fueron desarrolladas por las universidades sin la asignación de recursos específicos por parte del Estado Nacional. En el caso particular de los Protocolos, recién a partir de la sanción de la Ley Micaela, se aprobó en 2019 una convocatoria para el “Desarrollo de Protocolos y/o Herramientas Institucionales para la prevención y la intervención ante situaciones de discriminación y/o violencias de género en el ámbito universitario". Los recursos para su financiamiento provinieron del Fondo Universitario para el Desarrollo Nacional y Regional del Ministerio de 
Educación de la Nación, que prevé trasferencias a las universidades nacionales para financiar gastos corrientes sin especificación de finalidad. De manera tal, que si bien se sancionó y adhirió formalmente a una Ley que obliga a las universidades a capacitar en la temática, no se contemplaron a la fecha fondos delimitados para tal fin.

\section{Compromiso con el Objetivo de Desarrollo Sostenible "lograr la igualdad de género y empoderar a todas las mujeres y las niñas". ¿Cuál es el papel de las universidades?}

En el plano internacional, el año 2015 estuvo signado por la aprobación por parte de la Asamblea General de las Naciones Unidas (NU) del documento "Transformar nuestro mundo: la Agenda 2030 para el Desarrollo Sostenible" (NU, 2015). El documento definió 17 Objetivos de Desarrollo Sostenible (ODS) de los que se derivaron 169 metas. Se determinaron, además, una serie de medios para su ejecución; el seguimiento y examen de los progresos obtenidos en el plano nacional, regional y mundial; y un llamado a la acción de las naciones en los siguientes quince años. Se reconoció que para alcanzar estos Objetivos y metas, cada país enfrenta desafíos específicos y dispone de diversos enfoques, visiones de futuro, modelos e instrumentos; sin embargo, se acordó que la consecución de la igualdad de género y el empoderamiento de todas las mujeres y las niñas, contribuiría decisivamente al progreso de todos ellos (NU, 2015).

La adopción del enunciado Objetivo tuvo su antecedente en la "Declaración del Milenio" (NU, 2000), que supo plasmar el compromiso de los Jefes de Estado y de Gobierno, de promover la igualdad entre los sexos y la autonomía de la mujer. Sin embargo, no expresó un reconocimiento pleno de la importancia de la transversalidad de la perspectiva de género en todos los Objetivos y metas, tal como se señaló en el marco de los ODS. La Agenda 2030 se propuso por tanto como un plan de acción, atento a la premisa de que el desarrollo sostenible no será posible sin sociedades pacíficas, justas e igualitarias.

La República Argentina suscribió la "Declaración del Milenio" y en septiembre de 2015 se adhirió a la Agenda 2030. A partir de entonces, definió la responsabilidad por parte del Consejo Nacional de Coordinación de Políticas Sociales (CNCPS) de la Presidencia de la Nación (Decreto 499/2017), de coordinar las ac- 
ciones necesarias para su efectiva implementación. Para ello, debería articular sus acciones con aquellos organismos internacionales cuyas competencias se relacionaran con los postulados de la enunciada Agenda; pudiendo conformar comités técnicos y grupos de trabajo, tanto sectoriales como territoriales, para el adecuado monitoreo, seguimiento y evaluación de los ODS establecidos. Según lo decretado, se presentó en julio de 2017 un informe en el que se recopilaron los avances del Gobierno en la implementación de la Agenda 2030 y se consignó el inicio de adaptación de los ODS en el nivel nacional, provincial y local; indicándose, además, la participación activa del sector privado empresarial, del académico y de la sociedad civil (CNCPS, 2017).

El reconocimiento del sector académico y de las universidades como actores relevantes en el alcance de las metas de los ODS adoptadas por el país, tuvo también su antecedente en las acciones desarrolladas en el marco de la suscripción de la "Declaración del Milenio". Como parte de éstas, en 2012 se llevó a cabo un relevamiento sobre las contribuciones de las universidades e institutos universitarios que conforman el Consejo Interuniversitario Nacional (CIN) al logro de los Objetivos de Desarrollo del Milenio (ODM). La iniciativa se llevó a cabo en el marco del acuerdo de cooperación establecido entre el CIN y el CNCPS en abril de 2011, y buscó visibilizar experiencias que aportaron a la transformación social, la paz, la equidad, el desarrollo humano inclusivo y la sustentabilidad medioambiental (CNCPS, 2012).

El establecimiento de la Agenda 2030 fue de la mano de una nueva convocatoria por parte del CNCPS, a iniciar un proceso de reflexión estratégica sobre la integración de los ODS en las instituciones universitarias. Como parte de las acciones relevadas en 2017, se informó la realización de reuniones con el Secretario Ejecutivo del CIN, con objeto de retomar el trabajo articulado en torno al seguimiento de los ODM. Así como la firma de un convenio marco de colaboración recíproca entre el CIN y el CNCPS; el avance en un primer acuerdo específico para la determinación de actividades conjuntas a través de las Comisiones de Extensión y Ciencia y Técnica del CIN; y en la firma de un convenio marco de cooperación con el Consejo de Rectores de las Universidades de Gestión Privada, que se suscribió en noviembre de 2018 (CNCPS, 2017).

En 2018, el CNCPS presentó el "Informe País", donde se proveyó información sobre los avances realizados en el conjunto de los 17 ODS. Para el seguimiento y análisis de los progresos, y a los fines de incorporar transversalmente la pers- 
pectiva de género, se convocó al Instituto Nacional de las Mujeres (INAM) ${ }^{(7)}$, el cual tuvo intervención en la definición y el tratamiento de las siguientes metas: eliminar todas las formas de violencia contra todas las mujeres y las niñas en el ámbito público y en el privado, incluidas la trata y la explotación sexual y otros tipos de explotación; reconocer y valorar los cuidados y el trabajo doméstico no remunerados mediante servicios públicos, infraestructuras y políticas de protección social, promoviendo la responsabilidad compartida; y asegurar la participación plena y efectiva de las mujeres y la igualdad de las oportunidades de liderazgo a todos los niveles decisorios de la vida política, económica y pública en el hogar y la familia. En este Informe, se hizo referencia a las universidades nacionales como destinatarias de las metas enunciadas, indicándose en estos casos el objetivo general de promover experiencias personales y colectivas de deconstrucción de la subjetividad patriarcal y de construcción de ética y política feminista a través de las herramientas de Educación Popular, que propiciaran el empoderamiento de las mujeres (CNCPS, 2018).

Como resultado de este proceso de relevamiento, se establecieron los indicadores de seguimiento de los ODS. En el caso de las metas definidas para alcanzar la igualdad de género, incluyeron: cantidad de femicidios ocurridos durante el período de un año en todo el territorio nacional; brecha de tiempo dedicado al trabajo no remunerado entre varones y mujeres por día; porcentaje de bancas ocupadas por mujeres en la Cámara de Diputados del Congreso Nacional, porcentaje de bancas ocupadas por mujeres en la Cámara de Senadores del Congreso Nacional, y proporción de mujeres en ocupaciones directivas. Las fichas requeridas para su seguimiento, fueron elaboradas por el INAM y el Ministerio de Salud y Desarrollo Social (CNCPS, 2019).

Tal como se advierte, pese a la existencia de antecedentes, de un marco de actuación formal y del reconocimiento del papel de las universidades en la implementación de las metas y alcance de los ODS, no fueron incluidas en esta instancia como actores en el proceso de construcción de los distintos indicadores en materia de género, ni en la recolección de la información. Su rol como se ma-

\footnotetext{
(7) Organismo descentralizado en la órbita del Ministerio de Desarrollo Social, encargado del diseño y propuesta de las políticas públicas de protección integral para prevenir y erradicar la violencia y discriminación contra las mujeres en los ámbitos en que desarrollen sus relaciones interpersonales (Decreto 698/2017).
} 
nifestara en los informes relevados, fue de destinatarias de los procesos de intervención del organismo responsable, en este caso el INAM.

En 2020, a partir de la asunción del nuevo Gobierno Nacional, se presentó un Informe donde se reafirmó el compromiso con los preceptos y fundamentos de la Agenda 2030. Se realizó un análisis de la situación inicial y de la evolución de los indicadores definidos durante la Administración previa (2015-2019). Se observó por un lado, la falta de adaptación de la herramienta (plataforma) que muestra los indicadores que resultaron de la adaptación en Argentina de los ODS, con aquella que permite evidenciar el panorama general del cumplimiento de los ODS en los distintos países. En términos del tratamiento del Objetivo de lograr la igualdad de género a nivel nacional, se advirtió un incremento sostenido en la cantidad de femicidios ${ }^{(8)}$; la falta de periodicidad en la implementación de la encuesta que mide el trabajo no remunerado y el uso del tiempo; un leve descenso en el porcentaje de bancas ocupadas por mujeres entre 2016 y 2018 en la Cámara de Diputados, y un incremento en la Cámara de Senadores; un escaso avance en la reducción de la brecha salarial de género; y la promoción de un ajuste en los presupuestos para combatir la violencia contra las mujeres (CNCPS, 2020). La intención de revisar los indicadores a la luz de las nuevas prioridades del Gobierno Nacional y la necesidad de intervenir para subsanar resultados como los señalados en el presente Informe, debiera contemplar el rol de las universidades como productoras y promotoras de políticas tendientes al efectivo logro de una sociedad más justa, inclusiva y equitativa.

\section{Articulación de las universidades con los organismos nacionales responsables de eliminar todas las formas de discriminación y violencia contra las mujeres y las niñas}

Es de destacar que el período de referencia de este artículo, que se centra en los años transcurridos desde 2015 al presente, estuvo impactado en la materia de género por la actuación de distintos organismos de alcance nacional. Por un lado, el Consejo Nacional de la Mujer, creado con el objetivo de concretar el compromiso asumido por el estado argentino al ratificar la adhesión a la "Con-

\footnotetext{
(8) En efecto, como informara la asociación civil “La Casa del Encuentro", se consignaron los siguientes casos de femicidios: en 2015, 286; en 2016, 290; en 2017, 295; en 2018, 273; y en 2019, de 299 (La Casa del Encuentro, 2020).
} 
vención sobre la eliminación de todas las formas de discriminación contra la mujer", y dependiente en sus inicios de Presidencia de la Nación (Decreto Nacional 1.426/1992). Posteriormente, se estableció su denominación como Consejo Nacional de las Mujeres (Decreto Nacional 326/2010), pasó a depender del Consejo Nacional de Coordinación de Políticas Sociales, y fue transferido luego a la órbita del Ministerio de Desarrollo Social. En el año 2017, los cometidos del CNM fueron asignados al Instituto Nacional de las Mujeres (INAM), que actuaría en esta instancia como organismo descentralizado en la órbita del citado Ministerio (Decreto 698/2017).

De lo expresado, se advierte que el Objetivo de lograr la igualdad de género requiere la adopción de medidas tendientes a la eliminación de todas las formas de discriminación y violencia(9) contra las mujeres y las niñas. Esta meta ha estado presente en la normativa y adhesión de nuestro país a distintos convenios e instrumentos de carácter internacional(10); y en particular, en la"Ley de protección integral para prevenir, sancionar y erradicar la violencia contra las mujeres en los ámbitos en que desarrollen sus relaciones interpersonales" (Ley № 26.485, 2009). Con el fin de cumplimentar esta Ley, el Consejo Nacional de Coordinación de Políticas Sociales informó el diseño, presentación y validación del “Plan Nacional de Acción para la Prevención, Asistencia y Erradicación de la Violencia contra las Mujeres 2017-2019" (Consejo Nacional de las Mujeres - Consejo Nacional de Coordinación de Políticas Sociales, 2016). La presentación del referido Plan estuvo a cargo del Consejo Nacional de las Mujeres (CNM), e incluyó dos ejes de actuación orientados a la prevención y atención integral de las mujeres en situación de violencia de género, "y tres ejes transversales que apuntaban a la formación en perspectiva de género en todos los niveles, un fuerte trabajo de articulación y coordinación institucional y un permanente monitoreo y evaluación de las políticas públicas" (CNM - CNCPS, 2016, p. 6). Si bien la Ley de protección integral a las mujeres (Ley $\left.N^{\circ} 26.485,2009\right)$ supo contemplar también la articu-

\footnotetext{
(9) La Convención Interamericana para Prevenir, Sancionar y Erradicar la Violencia contra la Mujer entiende por violencia contra la mujer cualquier acción o conducta, basada en su género, que cause muerte, daño o sufrimiento físico, sexual o psicológico a la mujer, tanto en el ámbito público como en el privado (OEA, 1994).
}

(10) Véase: Ministerio de las Mujeres, Géneros y Diversidad (2020). 
lación y coordinación con las universidades para el desarrollo de estas acciones, no fueron mencionadas en las distintas instancias de elaboración del Plan.

Seguidamente, atendiendo a las funciones asignadas al INAM, que incluían la coordinación y articulación con organismos a nivel nacional, provincial, de la Ciudad Autónoma de Buenos Aires y municipal, del desarrollo de programas de promoción, capacitación, actualización y perfeccionamiento destinados a la eliminación de la violencia y discriminación de la mujer (Decreto 698/2017); se informó en 2019, la capacitación de 4.446 personas de la Administración Pública Nacional y de 4.468 personas del Poder Judicial, en tanto que en septiembre de ese año se dio inicio al primer curso en la Dirección de Recursos Humanos de la Cámara de Diputados (Jefatura de Gabinete de Ministros, 2020). En su reporte de ejecución, el INAM informó además, sobre diversas actuaciones que involucraron al sistema universitario nacional. Se señaló la puesta en marcha de campañas de difusión; la suscripción de convenios; la ejecución de talleres y capacitaciones; y la asistencia técnica para el diseño y elaboración de cursos de sensibilización y formación en la temática de género destinados a docentes, no docentes y estudiantes (INAM, 2019).

Una vez más, el rol asignado a las universidades públicas argentinas pareciera desconocer sus aportes en términos de la visibilización por un lado, de diferentes tipos y manifestaciones de violencia y discriminación; del reconocimiento de distintos actores afectados a ella, no sólo mujeres y niñas; de la promoción de actividades tendientes a su prevención, atención, sanción y erradicación; y a la contribución de la institucionalización y transversalización de la perspectiva de género.

\section{Institucionalización y transversalización de las políticas de género en el marco de las universidades nacionales}

En Argentina, pese al compromiso asumido por el Gobierno con el logro de la igualdad de género y empoderamiento de todas las mujeres y niñas, y de la puesta en marcha de las diversas iniciativas orientadas a su concreción, quedan importantes retos por enfrentar. En tal sentido, la educación constituye un instrumento central para empoderar a las personas, y a su vez, representa un indicador de las desigualdades y disparidades de género. En el caso de las universidades argentinas, esta situación se pone de manifiesto en distintas esferas, entre 
ellas, en el acceso a ciertas carreras, y en la representación en las instancias de gobierno y toma de decisiones ${ }^{(11)}$.

Las universidades argentinas públicas, afectadas desde sus inicios por dificultades para alcanzar la plena igualdad de derechos y oportunidades, por vivencias cotidianas de discriminación y violencias ligadas al género y la sexualidad, supieron visibilizar y reflexionar en torno a estas cuestiones. Impulsaron la promoción de espacios de género, fundamentales para la generación de un conocimiento crítico sobre las distintas formas de desigualdad; y la incorporación de las temáticas de género en los planes y programas de estudio del nivel superior. Alentaron la institucionalización y transversalización de la perspectiva de género, que atravesó las distintas dimensiones de la vida universitaria, más allá de sus funciones sustantivas -la investigación, la extensión y la formación-, adentrándose en sus prácticas, en sus leyes y en sus estructuras.

En concordancia con lo expresado por la Asamblea General de las Naciones Unidas, la incorporación sistemática de una perspectiva de género es crucial y supone aún un desafío (Naciones Unidas, 2015). Pero es posible avanzar en pos de ella a través de acciones en línea con las cuales, las universidades públicas argentinas, en el período de referencia del presente artículo, han realizado un significativo aporte.

En primer lugar, contribuyendo al desarrollo de datos, estadísticas e indicadores oportunos y relevantes con enfoque de género. Estos instrumentos brindan conocimiento sobre los roles y la distribución de los derechos de mujeres y hombres en las sociedades, y son una herramienta fundamental para obtener evidencia y monitorear las causas y efectos de la desigualdad de género. El trabajo de coordinación y sistematización iniciado por RUGE en relación con los programas, herramientas y actividades de su área de competencia, promovidas desde las instituciones que la integran, dio cuenta de una aportación en este sentido.

En segundo lugar, incorporando la perspectiva de género en la legislación universitaria como una base legal para impulsar cambios que garanticen la equidad. Frente a la persistencia de disparidades en el acceso a cargos y carreras en el ámbito de la Educación Superior, instituciones como la Universidad Nacional de Río Negro, la Universidad Nacional de La Rioja, la Universidad Nacional de San Martín, la Universidad de Buenos Aires y la Universidad Nacional de Ge-

(11) Véase: Secretaría de Políticas Universitarias (2020). 
neral Sarmiento, avanzaron desde 2017, bajo distintas formas, en el reconocimiento e incorporación de la paridad de género en su normativa. La Universidad Nacional de Cuyo fue la primera en el país en implementar la paridad, no sólo para las listas de los órganos colegiados, sino también para los cargos ejecutivos, es decir, para el Rectorado y Decanatos. La paridad de género representa un aspecto relevante a la hora de alcanzar la equidad; pero el establecimiento de esta norma debe ser acompañado por una política integral de género fomentada desde las propias universidades, que posibilite y promueva las condiciones para su aplicación.

En tercer lugar, impulsando la sensibilización en temas de género. La sanción de la"Ley Micaela de capacitación obligatoria en género para todas las personas que integran los tres poderes del Estado"; la formalización de su adhesión por parte de las instituciones que conforman el CIN; $y$ la puesta en marcha de las distintas actividades de capacitación por parte de las universidades nacionales; representan un nuevo aporte en torno a esta propuesta. Es de destacar que la implementación de las capacitaciones obligatorias en la temática de violencia machista, fue uno de los puntos ineludibles que se afirmaron el 3 de junio 2015, como parte del camino hacia "Ni Una Menos".

En cuarto lugar, promoviendo acciones tendientes a la eliminación de todas las formas de discriminación y violencia contra todas las mujeres y las niñas. En este aspecto, es de destacar que el reclamo iniciado en el año 2015 a través de "Ni Una Menos", siguió demostrando al presente una respuesta insuficiente. En efecto, el período transcurrido desde la masiva movilización que se centrara en la denuncia del femicidio como un problema estructural, dio cuenta de un incremento en su registro en todo el país. Las universidades nacionales incorporaron indudablemente la agenda contra la violencia de género, impulsaron la sanción de protocolos de actuación, la creación de dispositivos de atención y de programas por la igualdad de géneros y contra las violencias sexistas. Esto supuso, además, la voluntad de intervenir a través de estos dispositivos sobre las interacciones cotidianas, los valores, normas, códigos culturales e imaginarios propios de la universidad con el objeto de subvertir las regulaciones sexo genéricas que traman el espacio universitario (Blanco, 2016).

Por último, coordinando iniciativas con organismos competentes en la temática de género. A modo de ejemplo, en 2020, la Red Interuniversitaria por la Igualdad de Género y Contra las Violencias firmó un convenio con la Iniciativa Spotlight, 
una campaña conjunta de la Unión Europea y las Naciones Unidas orientada a eliminar todas las formas de violencia contra las mujeres y las niñas; que tendrá como objeto profundizar y fortalecer las políticas institucionales para su erradicación del sistema universitario.

La puesta en marcha de estas medidas supone un reconocimiento de la agenda internacional en materia de género, de los compromisos asumidos a nivel nacional, y de las demandas de la sociedad en la que las universidades están insertas. Representa además, una trasformación de sus comunidades que trasciende sus propias fronteras.

\section{Nuevos compromisos gubernamentales en materia de género}

A partir de la asunción del nuevo Gobierno en diciembre de 2019, se buscó ampliar el alcance, funciones y competencias del Estado Nacional en materia de género y diversidad, para lo cual, se suprimió el Instituto Nacional de las Mujeres y se estableció la creación del Ministerio de las Mujeres, Géneros y Diversidad (Decreto 7/2019).

El reconocimiento de la jerarquía de ministerio; y su compromiso con los derechos de las mujeres y diversidades, frente a toda forma de discriminación y violencia, constituye una buena señal en términos de la construcción de una sociedad más igualitaria, que promueva la autonomía integral de todas las personas, con independencia de las diversas orientaciones sexuales, identidades o expresiones de género. La labor de visibilización e inclusión de estas diversidades, fue parte de los aportes realizados por las universidades en la presente coyuntura. Por otro lado, la propuesta de trabajar en articulación con un Consejo Asesor ad honorem, que incluya activistas feministas de la diversidad sexual y de las identidades de género de reconocida trayectoria en diversos ámbitos, supone también un posible signo de cambio. Es de destacar que, si bien este Consejo actuará como órgano de consulta, se instituye como un espacio de diálogo y promoción de iniciativas en el que se reconoce la presencia de destacadas referentes del ámbito académico. Además, como parte de las acciones impulsadas desde el Ministerio, se presentó en junio de 2020, un nuevo Plan Nacional de Acción contra las violencias por motivos de género, donde se informó la intención de realizar el seguimiento de avances y monitoreo del Plan, en colaboración con universidades nacionales y con el Ministerio de Ciencia, Tecnología e Innovación 
de la Nación (Ministerio de las Mujeres, Géneros y Diversidad, 2020). Finalmente, en un contexto en el que no se ha aprobado aún el Proyecto de Ley de Presupuesto Nacional 2020, el Jefe de Gabinetes reasignó fondos al Ministerio de las Mujeres, Géneros y Diversidad, a fin de atender al diseño, ejecución y evaluación de las políticas públicas nacionales en materia de género, igualdad y diversidad (DECAD-2020-2-APN-JGM).

\section{CONCLUSIONES}

El compromiso asumido por la República Argentina con el logro de la igualdad de género y el empoderamiento de todas las mujeres y las niñas está en línea con la legislación vigente, y la ratificación del país de tratados e instrumentos no vinculantes internacionales. La adhesión a la“Agenda 2030 para el Desarrollo Sostenible" significó además, el establecimiento de organismos de alcance nacional encargados de coordinar las acciones necesarias para su efectiva implementación y de asistirlo en el tratamiento del Objetivo. En este sentido, las acciones emprendidas desde el Gobierno en el período comprendido entre los años 2015 y 2019 mostraron una escasa efectividad. Esto se manifestó, principalmente, en un presupuesto menguante y en la dificultad de brindar seguimiento a las acciones emprendidas. Si bien se reconoció a las universidades como actores relevantes en materia de política de género, su participación tuvo el carácter de destinatarias de las iniciativas promovidas desde estos organismos y una muy escasa participación en el diseño e implementación de las políticas públicas.

Frente a un escenario que puso de manifiesto la insuficiencia de las medidas adoptadas hasta el momento para atender a la erradicación de la discriminación y violencias de género en todos sus tipos y modalidades, y contemplando el Objetivo definido por el Gobierno actual, resulta fundamental reconocer las contribuciones de las universidades. Por un lado, en términos de la labor impulsada "desde abajo hacia arriba" por los movimientos de mujeres y feministas para transformar las estructuras patriarcales dominantes en sus propias instituciones; $y$ a su vez, desde las acciones impulsadas como parte de su trabajo en red, que ha fortalecido la institucionalización y transversalización de la perspectiva de género en el sistema universitario. Por tanto, se debiera contemplar su inter- 
vención en la elaboración del diagnóstico de la problemática vigente, para avanzar en la promoción de indicadores y estrategias eficientes para atender a la solución de las metas comprendidas en el Objetivo, y en la asignación de recursos específicos para ponerlas en marcha, así como considerar su participación en el seguimiento y evaluación de las diversas acciones. Si bien la reafirmación del compromiso del Gobierno con el logro de la igualdad es alentadora, resulta necesario aún reconocer plenamente el rol de las universidades argentinas públicas en la construcción y promoción de políticas tendientes a la consecución de una sociedad más igualitaria, base del desarrollo sostenible.

\section{REFERENCIAS}

Acuerdo Plenario № 1076 de 2019 por el cual se establece la adhesión del Consejo Interuniversitario Nacional a la Ley N ${ }^{\circ} 27.499$ - Ley Micaela. 8 de abril de 2019.

Anzorena, C. (2008). La participación de las mujeres en el proceso de formación del estado nacional en Argentina de finales del siglo XIX. Reflexiones desde una perspectiva de género. Revista lberoamericana de Educación. Núm. 45/2. https://rieoei.org/historico/deloslectores/2103Anzorena.pdf

Barrancos, D. (2012). Los estudios feministas en el sistema académico argentino. En: Ávila García, V. y Suárez, P. (Comps.). Entre mujeres te veas: las academias y los estudios feministas en México, Argentina, Venezuela y España pp.69-97. Palabra de Clío.

http://www.palabradeclio.com.mx/src pdf/Ent1513465628.pdf

Blanco, R. (2016). Más allá de los protocolos contra las violencias de género. Bordes. https://revistabordes.unpaz.edu.ar/mas-alla-de-los-protocolos-contra-las-violenciasde-genero/

Bustelo, N. (2018). Todo lo que necesitás saber sobre la reforma universitaria. Paidós.

Consejo Nacional de Coordinación de Políticas Sociales (2012). Objetivos de desarrollo del milenio. Contribuciones de las universidades e institutos universitarios que conforman el Consejo Interuniversitario Nacional. CNCPS. https://www.undp.org/content/dam/argentina/Publications/ODM\%20Argentina/undp ar 2013 \%200DMyUNIV2012.pdf 
Consejo Nacional de Coordinación de Políticas Sociales (2017). Informe voluntario nacional. Argentina. CNCPS. https://www.argentina.gob.ar/sites/default/files/politicassociales-publicaciones-informe-voluntario-nacional-ods-argentina-julio-2017 $1100 . p d f$

Consejo Nacional de Coordinación de Políticas Sociales (2018). Objetivos de desarrollo sostenible - informe país - Argentina 2018. CNCPS. https://www.argentina.gob.ar/sites/default/files/informe-ods-todo.pdf

Consejo Nacional de Coordinación de Políticas Sociales (2019). Metadata de los indicadores de seguimiento de los ODS. Agenda 2030. Argentina. CNCPS. https://www.argentina.gob.ar/sites/default/files/metadata_25_09_19.pdf

Consejo Nacional de Coordinación de Políticas Sociales (2020). Segundo informe voluntario nacional. Argentina 2020. CNCPS. https://www.odsargentina.gob.ar/public/documentos/seccion_publicaciones/ivn_2020.pdf

Consejo Nacional de las Mujeres - Consejo Nacional de Coordinación de Políticas Sociales (2016). Plan nacional de acción para la prevención, asistencia y erradicación de la violencia contra las mujeres (2017-2019). CNM CNCPS.

https://www.argentina.gob.ar/sites/default/files/consejo_nacional_de_mujeres_plan_nacional_de_accion_contra_violencia_genero_2017_2019.pdf

Constitución de la Nación Argentina. 15 de diciembre de 1994. Publicada en el Boletín Oficial N² 28057.

Convención Interamericana para Prevenir, Sancionar y Erradicar la Violencia contra la Mujer (Convención de Belém do Pará). Organización de los Estados Americanos. 09 de junio de 1994.

Decreto 1.426 de 1992 por medio del cual se establece la creación del Consejo Nacional de la Mujer. 07 de agosto de 1992. Boletín Oficial, 13/08/92. DN19920001426.

Decreto 326 de 2010 por medio del cual se establece que el Consejo Nacional de la Mujer pasará a denominarse "Consejo Nacional de las Mujeres". 08 de marzo de 2010. Boletín Oficial N³1859.

Decreto 499 de 2017 por medio del cual se establece al Consejo Nacional de Coordinación de Políticas Sociales como organismo responsable de coordinar las acciones necesarias para la efectiva implementación de la "Agenda 2030 para el Desarrollo Sostenible". 12 de julio de 2017. e. $13 / 07 / 2017$ N$^{\circ} 50215 / 17$ v. 13/07/2017. 
Decreto 698 de 2017 por medio del cual se crea el Instituto Nacional de las Mujeres. 05 de septiembre de 2017. e. 06/09/2017 N 66011/17 v. 06/09/2017.

Decreto 7 de 2019 por medio del cual se crea el Ministerio de las Mujeres, Géneros y Diversidad. 10 de diciembre de 2019. e. 11/12/2019 N $96118 / 19$ v. 11/12/2019.

Decisión Administrativa 2 de 2020 por medio de la cual se modifica la distribución del Presupuesto General de la Administración Nacional para el Ejercicio 2020. 13 de enero de 2020. Jefe de Gabinete de Ministros. e. $14 / 01 / 2020 \mathrm{~N}^{\circ} 1796 / 20$ v. 14/01/2020.

Gómez, C. (2019). Las condiciones de mi sexo: mujeres científicas argentinas frente a la memoria masculinizada. Revista de Investigación del Departamento de Humanidades y Ciencias Sociales - UNLaM, núm. 16 (8). https://rihumso.unlam.edu.ar/index.php/humanidades/article/view/179/pdf

Instituto Nacional de las Mujeres (2019). Reporte de ejecución del plan nacional de acción para la prevención, asistencia y erradicación de la violencia contra las mujeres 2017-2019. Ley 26.485. INAM. https://trello.com/b/GjiiMl3V/monitoreo-del-plan-nacional-gob-abierto

Jefatura de Gabinete de Ministros (2020). Memoria detallada del estado de la nación 2019. JGM. https://www.argentina.gob.ar/sites/default/files/Memoria2019.pdf

La Casa del Encuentro (2020). Observatorio de femicidios en Argentina Adriana Marisel Zambrano. http://www.lacasadelencuentro.org/femicidios03.html

Ley $N^{\circ} 1.420$ de 1884 por la cual se establece la Ley de Educación. 26 de Junio de 1884. Publicada en R.N.1882/84.

Ley N² 26.485 de 2009 por la cual se sanciona la Ley de protección integral para prevenir, sancionar y erradicar la violencia contra las mujeres en los ámbitos en que desarrollen sus relaciones interpersonales. 1 de abril de 2009. Boletín Oficial № 31632.

Ley № 27.499 de 2019 por la cual se establece la Ley Micaela de capacitación obligatoria en género para todas las personas que integran los tres poderes del Estado. 10 de enero de 2019. e. 10/01/2019 № 1607/19 v. 10/01/2019. 
Ministerio de las Mujeres, Géneros y Diversidad (2020). Plan nacional de acción contra las violencias por motivos de género (2020-2022). Ministerio de las Mujeres, Géneros y Diversidad. https://www.argentina.gob.ar/sites/default/files/plan_nacional_de_accion_2020_2022.pdf

Ni Una Menos (2015). Manifiesto 3 de junio de 2015. Ni Una Menos. http://niunamenos.org.ar/manifiestos/3-de-junio-2015/

Ordenanza del Consejo Superior de la Universidad Nacional del Comahue № 1572. Por medio de la cual se aprueba el Protocolo de intervención institucional ante denuncias por situaciones de violencia sexista en el ámbito de la Universidad Nacional del Comahue. 5 de junio de 2014.

Palermo, A. (2005). Mujeres profesionales que ejercieron en Argentina en el siglo XIX. Convergencia. Revista de Ciencias Sociales. vol. 12, núm. 38, pp. 59-79. https://www.redalyc.org/pdf/105/10503803.pdf

Palermo, A. (2006). El acceso de las mujeres a la educación universitaria. Revista Argentina de Sociología. vol. 4, núm. 7, pp. 11-46. https://www.redalyc.org/pdf/269/26940702.pdf

Proyecto de Ley de 2017 por el cual se trata el Proyecto de capacitación obligatoria en la temática género y violencia contra las mujeres, para todas las personas que integran los tres poderes del Estado - Ley Micaela. 19 de abril de 2017. Expediente 1776-D-2017.

Resolución 70/1 de 2015. Asamblea General de las Naciones Unidas. Transformar nuestro mundo: la Agenda 2030 para el Desarrollo Sostenible. 25 de septiembre de 2015.

Resolución 55/2 de 2000. Asamblea General de las Naciones Unidas. Declaración del Milenio. 8 de septiembre de 2000.

RUGE (2018). Pronunciamiento de la red universitaria de género en el centenario de la reforma universitaria - 2018. CIN. https://www.cin.edu.ar/pronunciamiento-de-la-red-universitaria-de-genero-en-el-centenario-de-la-reforma-universitaria/

Secretaría de Políticas Universitarias (2020). Mujeres en el sistema universitario argentino 2018-2019. SPU.

https://www.argentina.gob.ar/sites/default/files/mujeres_en_el_sistema_universitario_argentino - estadisticas 2018-2019.pdf

Torlucci, S.; Vazquez Laba, V.; Pérez Tort, M. (2019). La segunda reforma universitaria: políticas de género y transversalización en las universidades. Revista científica de la red de carreras de Comunicación Social - UNLP. núm. 9. https://doi.org/10.24215/24517836e016 


\section{BIONOTA}

Mariana Laura Solans. Lic. en Relaciones Internacionales en la USAL (2004). Mg. en Ciencia Política y Sociología en la FLACSO (2014). Ha realizado estudios de posgrado en Planeamiento y Gestión de la Educación, en Bioeconomía y en Ecología Integral. Directora de Posgrado y Capacitaciones en la Universidad Nacional de los Comechingones (UNLC), Argentina.

Correo electrónico: msolans@unlc.edu.ar https://orcid.org/0000-0002-9674-886X 